\title{
The Introduction of a New Robot for Assistance in Ophthalmic Surgery
}

\author{
M. A. Nasseri ${ }^{1}$, M. Eder ${ }^{2}$, S. Nair ${ }^{2}$, E. C. Dean ${ }^{2}$, M. Maier ${ }^{3}$, D. Zapp ${ }^{3}$, C. P. Lohmann ${ }^{3}$ and A. Knoll ${ }^{2}$
}

\begin{abstract}
This paper introduces the design and development of a new robotic system to assist surgeons performing ophthalmic surgeries. The robot itself is very compact and similar to an average human hand in size. Its primary application is intraocular micromanipulation in order to overcome the existing challenges in treatment of diseases like Retinal Vein Occlusion (RVO). The novel hybrid mechanism designed for this robot allows microscale motions and is stable in the presence of vibrations common in operation room $(\mathrm{OR})$. The robotic system can be easily integrated into standard operation rooms and does not require modification of conventional surgical tools. This compact microsurgical system is suitable for mounting on the patient's head and thereby, solves the problem of patient motion. The compatibility of the robotic system with a real world surgical setup was evaluated and confirmed in this work.
\end{abstract}

\section{INTRODUCTION}

Inherent scale and fragility of the human eye anatomy makes retinal surgery an extremely difficult procedure. Some treatments such as, retinal vein cannulation for occluded retinal vessels are currently not feasible due to technological and physiological limitations. Over 16 million people worldwide suffer from Retinal Vein Occlusion (RVO) [1]. RVO develops when a clot is formed in one of the retinal arteries or veins. These vessels have a cross section diameter of $80 \mu \mathrm{m}$. A promising treatment is the injection of clot-dissolving drugs such as, tissue Plasminogen Activator (tPA) directly into the affected vessel [2]. Wei et al. suggested a method using stenting as a surgical solution for Central Retinal Vein Occlusion (CRVO) [3]. However, the surgeon's movement skills are limited in performing tasks such as, accurately locating the tools and steadily holding it for at least 30 seconds. The tremor of the surgeon's hand is in the range of $108 \mu \mathrm{m}$ [4]. Therefore, it is practically impossible for the surgeon to accomplish such a high-precision procedure. Robots exhibiting high geometric accuracy, stability and precision motion at variable scales are suitable choices for performing precise retinal surgeries. Guerrouad and Jolly introduced one of the first ocular robotics systems in 1989, the "stereotaxical micromanipulator (SMOS)", which was a spherical micromanipulator mounted on a 3D stage to allow 6 Degrees Of Freedom (DOF) motion [5]. In 1997

\footnotetext{
*This work was supported by TUM Graduate School of Information Science in Health

${ }^{1}$ M. Ali Nasseri is with Graduate School of Information Science in Health, Technische Universität München nasseri at in.tum.de

${ }^{2}$ M. Eder, S. Nair, E.C. Dean and A. Knoll are with the Department of Robotics and Embedded Systems, Institut für Informatik, Technische Universität München ederma, dean, nair, knoll at in.tum. de

${ }^{3}$ M. Maier, D. Zapp and C. P. Lohmann are with the Augenklinik rechts der Isar, Technische Universität München mathias.maier, daniel.zapp, c.lohmann at mri.tum.de
}

Charles et al. described their eye surgery robot called RAMS which is a $6 \mathrm{DOF}$ master-slave serial manipulator with 10 micron precision [7]. Wei et al. addressed a hybrid twoarmed microsurgical device based on hexapods [8]. Teiga et al. proposed similar kinematics for their robot [6]. Riviere group's approach is a hand-held surgical tool with the ability of active tremor cancellation [9]. Steady-hand is a robot assisting ophthalmic surgeons developed by Taylor's group at the Johns Hopkins University [10]. This robot is a cooperative surgical device which increases the precision and filters the hand tremors of the surgeon. Ueta et al. at Tokyo University proposed a spherical device for ophthalmic surgery in 2009 [11]. The Pooerten group from Katholieke Universiteit Leuven [12] and Meenik et al. from TU Eindhoven are two other groups working in the same area. The latter developed a fixed Remote Center of Motion (RCM) mechanism for retinal surgery which is mounted on an operation table [14].

Any typical ophthalmic surgical procedure demands a compact robotic system capable of micromanipulation and the ability to compensate the surgeon's hand tremor. The system integration into conventional operation theaters should require minimum effort without modification to the existing $\mathrm{OR}$, guarantee the maximum safety and manipulate conventional surgical tools. The integration of all these requirements in a single system has not yet been realized. This work proposes a robotic system which fills these mechanical design gaps and introduces a compact micro surgical masterslave robotic system. This system can also be fixated on the patient's forehead, which addresses key clinical requirements such as, patient's relative head motion. The improvements of the proposed system in comparison to the state of the art are: compactness, portability and varied OR compatibility. Furthermore, this system allows microscale motions and it

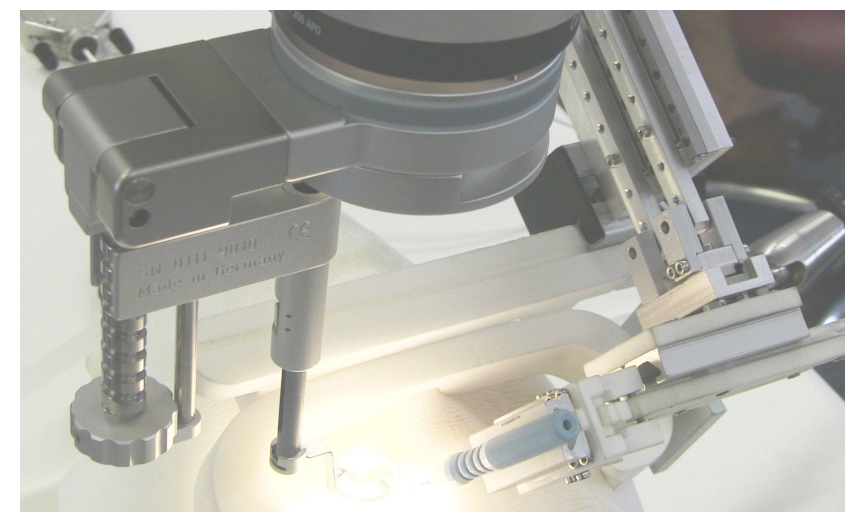

Fig. 1. Operation room compatibility evaluation, robot-goggle mounted on a dummy head under ophthalmic surgery microscope. 
is stable in the presence of vibrations common in the OR.

\section{The Developed Robot}

A hybrid parallel-serial robot comprising of prismatic piezo actuators was designed and developed. The kinematics consists of four serial segments; two parallel coupled joint elements and one prismatic plus one optional revolute joint in the end effector which collectively enables 6DOF tool motion.

\section{A. Parallel Coupled Joint Mechanism (PCJM)}
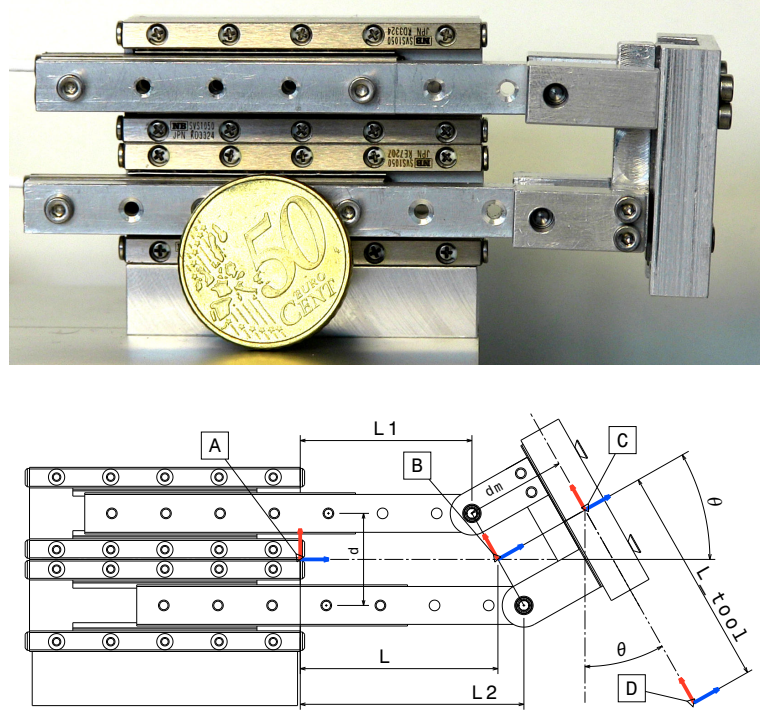

Fig. 2. Parallel Coupled Joint Mechanism (PCJM) developed to be used as the joint elements of ophthalmic surgery robot

The novel element designed in this work is a parallel coupled joint mechanism (see Fig.2). In this mechanism the differential displacement of two translational motions is converted to one translation and one rotation. $L 1$ and $L 2$ are linear displacements of each prismatic joint with distance $d$ from each other. $L$ and $\theta$ are linear and angular displacements of the mechanism $d_{m}$ is the length of the end effector and $L_{\text {tool }}$ is the length of the tool from gripper to tool tip. Equations in (1) represent the simplification of the mechanism by mapping $L 1$ and $L 2$ to $L$ and $\theta$ respectively

$$
L=\frac{L 1+L 2}{2}, \quad \theta=\arctan \left(\frac{L 2-L 1}{d}\right)
$$

Every parallel coupled joint simplifies to a prismatic and a revolute joint in serial. For instance, from Fig.2 and by considering $T_{A}^{D}=T_{A}^{B} T_{B}^{C} T_{C}^{D}$, the homogeneous transformation matrix of the tool tip with respect to the base is:

$$
T_{A}^{D}=\left[\begin{array}{cccc}
\cos \theta & -\sin \theta & 0 & \overbrace{L-L_{\text {tool }} \sin \theta+d_{m} \cos \theta}^{F . K} \\
\sin \theta & \cos \theta & 0 & L_{\text {tool }} \cos \theta+d_{m} \sin \theta \\
0 & 0 & 1 & 0 \\
0 & 0 & 0 & 1
\end{array}\right]
$$

The simplified calculation of homogeneous transformation matrices, $\theta$ and $L$ are substituted by $L_{1}$ and $L_{2}$. With these assumptions, any configuration consisting of PCJM is simplified and can be analyzed as a simple serial manipulator.

By considering the forward kinematics equations derived from the matrix in (2) and the $2 \mathrm{D}$ position of Point $\mathrm{D}$ in Fig.2, a unique dependency of L, $\theta$ and $L_{t o o l}$ is observed. This dependency enables pivoting the tool around point $\mathrm{D}$ as a RCM. In L1 and L2 domain, this situation is interpreted as the dependent differential displacement of two parallel prismatic joints.

As described in the previous paragraphs, the PCJM is similar to a serial pair of prismatic and revolute joints. The advantages of this mechanism over standard serial prismatic-revolute configuration are as follows: 1) Stiffness: Evidently, the parallel pair provides higher stiffness of the end effector against mechanical disturbances than a serial configuration [13]. 2) Precision: Piezo prismatic actuators with sub nano precision are among the most accurate available actuators. 3) Output force: For rotation and translation, both actuators are active simultaneously and introduce force to the system. In the serial setup, joint $n$ should carry the weight of joint $n+1$. Therefore, it needs to allocate a portion of its output force. While in the proposed configuration this effect is not seen. 4) Adjustable RCM: Any physical and virtual point that is in angular reach of the system can be defined as the pivoting point of the end effector or attached tool. This point can be changed or moved during motion. Each PCJM can provide a planar RCM. Therefore by having a serial configuration of two PCJMs, spatial RCM is satisfied.

A parallel coupled joint with piezo electric actuators ${ }^{1}$, low friction and precise mechanical components, sub micron optical encoders and customized controller was developed. The properties of this mechanism were measured as follows: dimensions: $94 \pm 28 \times 33.5 \times 18.5 \mathrm{~mm}$, weight: $150 \mathrm{~g}$, linear travel range: $\pm 28 \mathrm{~mm}$, angular rotation: $\pm 58.734^{\circ}$, linear precision: $1 \mu \mathrm{m}$, angular precision: $3.369 \times 10^{-3 \circ}$, maximum output force: $4.97 \mathrm{~N}$, maximum linear velocity: $17.5 \frac{\mathrm{mm}}{\mathrm{s}}$ and maximum angular velocity: $63.56_{\frac{s}{5}}^{\circ}$.

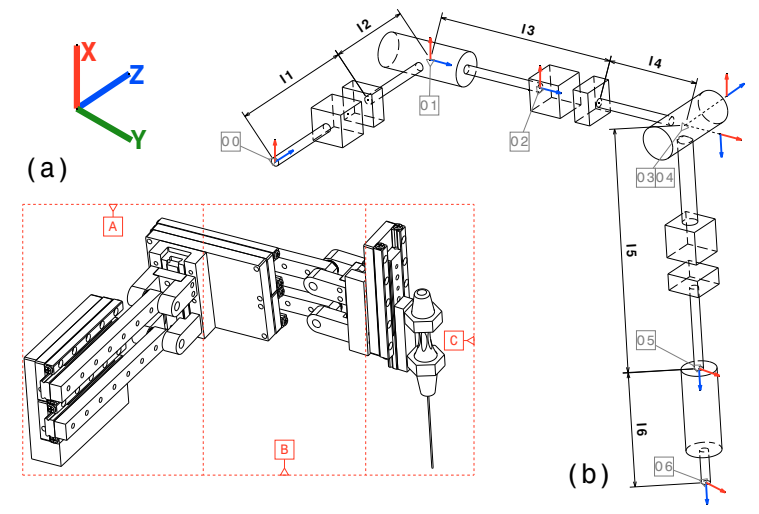

Fig. 3. (a) Serial robot: A and B are parallel coupled joint elements. C is the tool gripper consisting of a prismatic actuator and an optional tool rotator, (b) relevant and simplified model of the serial robot

\footnotetext{
${ }^{1}$ SmarAct linear positioners SLC-1750, SmarAct GmbH, Germany
} 


\section{B. Serial Robot}

Fig.3.(a) represents a serial configuration to perform 6 DOF manipulation. This robot consists of 3 serial segments, two PCJM elements and the tool gripper comprising of a prismatic actuator for tool translation and an optional revolute actuator for tool rotation. In the previous subsection it has been proved that the PCJM can be replaced by a prismatic and a revolute joint to simplify the analysis. In Fig.3.(b) the simplified model of the 6DOF robot is shown. Based on the simplified model and the Denavit-Hartenberg parameters, the robot is analyzed as a standard serial manipulator.

1) Singularities: To find the singular configurations of the robot, the Jacobian matrix was analyzed. The robot is in singular configuration only if the distance between two prismatic actuators is zero. So inside the working space of the robot the Jacobian matrix is full rank and consequently there are no singularities.

2) Working Space: Considering the data from paragraph II.A, the working volume of the end effector or the tool tip of the developed robot is limited to a $28 \times 28 \times 28 \mathrm{~mm}$ box. Authors believe that this working volume is sufficient for intraocular manipulation [16] but it can also be extended by using actuators with more travel ranges. And the angular movement of the tool is limited to $\pm 73^{\circ}, \pm 73^{\circ}$ and $360^{\circ}$ around $X, Y$ and $Z$ axes, respectively.

3) $3 D$ Variable RCM: Similar to RCM determination for a single joint, utilizing PCJM in 6DOF robot enables variable RCM in 3D space. Any virtual or physical points in space (excluding the singular configuration) can be defined as a pivoting point. During the motion this point is able to move. The importance of this specification for ophthalmic surgery is that the surgeon can move the eyeball by changing the position of RCM during the surgery and continue the operation. In conventional manual ophthalmic surgery this situation helps surgeons to see the different areas of retina through the microscope.

\section{ROBOT FOR OPHTHALMIC SURGERY}

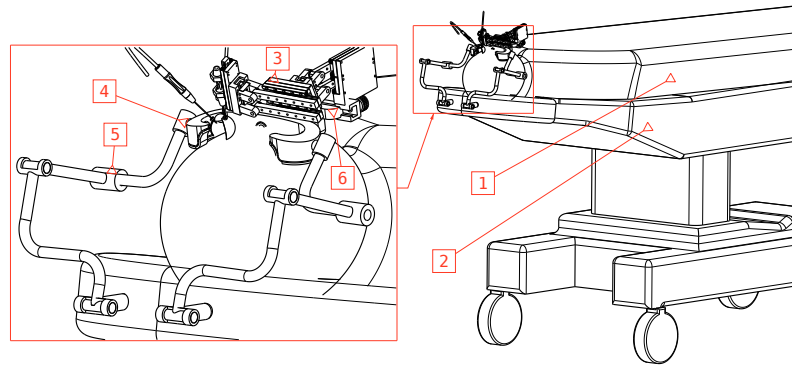

Fig. 4. Ophthalmic operation room; 1: patient, 2: operation table, 3: robot, 4: mounting goggle, 5: goggle arms, 6: robot slider

Referring back to the question of finding a solution to complex ophthalmic procedures such as, vein occlusion, the developed mechanism is a promising tool for performing micro injection into the retinal vein. Two remaining questions are how to mount the robot and how does the surgeon control the robot. This robot is used as a surgical tool which the surgeon employs for a period of 30 seconds up to a few minutes during the ophthalmic surgery to inject into the retinal vessel.

\section{A. The mounting mechanism of the robot}

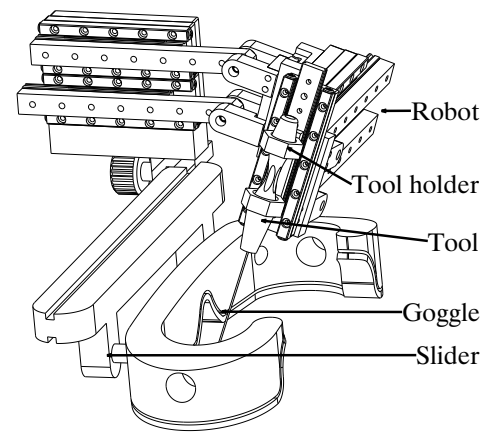

Fig. 5. Mounting mechanism of the robot with goggle and slider

The mounting mechanism is designed in a way that the surgeon can intuitively bring the robot on the patient's eye when needed and after the procedure it can be simply moved away and parked under the operation table. By means of this method the patient's head is fixed for the duration of t-Pa injection or any similar operation. Fig.4 and 5 illustrate the mounting mechanism. It consists of a slider mounted on a goggle which uses two arms with 4 DOF to be fixed on the patient's forehead. The robot is placed on the slider which enables the surgeon to adjust the robot in a suitable position. This way of mounting allows fixing the base of the robot with respect to the patient's head which thereby addresses an important problem of patient's head motion. Having a stable patient head posture is an important clinical requirement for ophthalmic surgery. Due to bulkiness and lack of relevant mounting structures, existing ophthalmic surgical robots can not be mounted on the patient's head.

\section{B. Operation Room Compatibility Evaluation}

The next step of the system validation is to check its compatibility to the operation room, operation table, microscope and other devices as well as the surgeon's accessibility to the operation area when the robot is mounted. These evaluations were conducted in the ophthalmology lab of $\mathrm{MRI}^{2}$ (see Fig.1). It was confirmed that the robot with the dimensions of $185 \times 44 \times 226 \mathrm{~mm}$ and a weight of $306 \mathrm{~g}$ is smaller than an average surgeon's hand, consequently it is compatible to the current ophthalmic surgery setup and it's suitable to be mounted on patient's head. Furthermore, according to the size of the robot and available areas on the goggle, using two robots simultaneously for the surgery is also possible. This will help the surgeon to carry out more complicated procedures such as, retinal artificial organ placement.

\footnotetext{
${ }^{2}$ Klinikum rechts der Isar der Technische Universität München. Available: http://www.augenklinik.med.tum.de/
} 


\section{Tool holder}

The tool holder of the current robot can hold conventional ophthalmic tools. It is designed to enable easy installation and change of different kinds of tools before and during the operation (see Fig.5).

\section{Clinical VAlidation}

The precision of the tool tip motion, from the global vision of the surgical site (using OR microscope), is measured to be less than $5 \mu \mathrm{m}$ in $[x, y]$ direction and $1 \mu \mathrm{m}$ in $[z]$ direction $([z]$ direction is decoupled and its precision is almost equal to the actuator precision). The achieved precision is substantially better than the one needed for vein cannulation, which is $20 \mu \mathrm{m}$ in all directions and better than the best recorded precision of a surgeon which is $108 \mu \mathrm{m}$. Also the output forces of the robot's end effector were measured as $4.97 \mathrm{~N}$ in $[x, y]$ and $2.84 N$ in $[z]$ directions are promising (refer to vitreoretinal forces measured in [15]) for the ophthalmic procedures.Based on the clinical motion data of the ophthalmic surgery during vitrectomy derived from [16], robot's motion in terms of positions and velocities was compared to the surgeon's motion during the operation. According to the observed data the robot with $28 \mathrm{~mm}$ travel range actuators is suitable for vein occlusion.

\section{CONCLUSIONS AND FUTURE WORKS}

The design and development of a robotic system for ophthalmic surgery has been described in this paper. Joint elements of the robot consisting of parallel piezo actuator pairs have been introduced which enables stable and precise motion with high output force. A serial configuration of these joints as an ophthalmic robot together with its simplified models have been shown. Furthermore an intuitive and practical way of mounting the robot on the patient's head for conducting the procedure has been presented. Finally, a brief report of the compatibility evaluation of the robot with operation room has been given. The fundamental advantages of our system are: Compatibility to OR: The size, the weight of the robot and the novel mounting mechanism make it a clinically compatible device. Patient's head fixture: The mounting mechanism containing goggle, slider and bars, makes the base of the robot fixed to the head of the patient, therefore there is no need to track the bed, patient and head motion. Stiffness: The robot comprising PCJM enables highest possible stiffness against environmental vibrations [13]. Variable RCM: Definition of the desired RCM point with simplified mathematical calculations is enabled by this setup. It allows the surgeon to define and even move the pivoting point of the tool during the procedure. This is important for the ophthalmic surgeons when they want to manipulate the eye ball to see different parts of the retina through the microscope and meanwhile perform the intraocular operation. Intuitive tool holder: The tool holder of this setup allows the surgeon to use the conventional ophthalmic tools. They can also change the tools during the surgery in an intuitive way. Safety: The characteristics of the piezo actuators used in this setup allow the surgeon to move the tool manually, in the opposite direction of insertion, by applying a force of around $5 N$.

Currently, the robot is being controlled by a developed PC interface. The authors plan to link the robot with a customized intuitive 6DOF master console to give the surgeons the possibility of performing complicated vitreoretinal procedures with high precision, dexterity and safety while keeping the operation similar to its conventional form.

\section{REFERENCES}

[1] S. Rogers et al., "The prevalence of retinal vein occlusion: pooled data from population studies from the US, Europe, Asia, and Australia," in Ophthalmology, vol. 117, no. 2, pp. 313-319, Feb. 2010

[2] L. Bynoe et al., "Retinal endovascular surgery for central retinal vein occlusion: initial experience of four surgeons," in Retina, Coral Springs, FL, USA, vol. 25, issue 5, pp. 625-632, Jul.-Aug. 2005

[3] W. Wei et al., "Enabling technology for microvascular stenting in ophthalmic surgery," in American Society of Mechanical Engineers (ASME): Journal of Medical Devices, vol 4, no. 1, Mar. 2010

[4] S. Sinch and C. Riviere, "Physiological tremor amplitude during retinal microsurgery," in Proceedings of the 28th IEEE Northeast Bioengineering Conference, PA, USA, pp. 171-172, Apr. 2002

[5] A. Guerrouad and D. Jolly, "Automatic analysis of weariness during a micromanipulation task by SMOS," in Proceedings of the IEEE Engineering in Medicine \& Biology Society 11th Annual International Conference, Seattle, DC, USA, pp. 906-907, Nov. 1989

[6] T. Nakano et al., "A parallel robot to assist vitreoretinal surgery," in International Journal of Computer Assisted Radiology and Surgery, vol. 4, issue 6, pp. 517-526, Nov. 2009

[7] S. Charles et al., "Dexterity-enhanced Telerobotic Microsurgery," in Proceedings of the IEEE International Conference on Advanced Robotics, Monterey, CA, USA, pp.5-10, Jul. 1997

[8] W. Wei et al., "Design and Theoretical Evaluation of Micro-Surgical Manipulators for Orbital Manipulation and Intraocular Dexterity," in Proceedings of the IEEE International Conference on Robotics and Automation, Roma, Italy, pp. 3389-3395, Apr. 2007

[9] W.T. Ang et al., "Active tremor compensation in microsurgery," in Proceedings of the 26th Annual International Conference of the IEEE Engineering in Medicine and Biology Society, Pittsburg, PA, USA, pp. 2738-2741, Sep. 2004

[10] A. Uneri et al., "New steady-hand eye robot with micro-force sensing for vitreoretinal surgery," in Proceedings of IEEE RAS EMBS International Conference on Biomedical Robotics and Biomechatronics, Tokyo, Japan, pp. 814-819, Sep. 2010

[11] T. Ueta et al., "Robot-assisted vitreoretinal surgery: Development of a prototype and feasibility studies in an animal model," in Ophthalmology, vol. 116, issue 8, pp. 1538-1543, 2009

[12] P. Caers et al., "Precision experiments on a comanipulated robotic system for use in retinal surgery," in Proceedings of the 2011 SCATh Joint Workshop on New Technologies for Computer/Robot Assisted Surgery, Graz, Austria, pp. 1-7, Jul. 2011

[13] T. K. Tanev, "Kinematics of a hybrid (parallel-serial) robot manipulator," In Mechanism and Machine Theory, vol. 35, pp. 1183-1196, 2000

[14] T. Meenink, "Vitreo-Retinal eye surgery robot: sustainable precision," $\mathrm{PhD}$ thesis, TU Eindhoven, Eindhoven, The Netherlands, ISBN:97890-386-2800-4, 2011

[15] A. S. Jagtap and C.N. Riviere, "Applied force during vitreoretinal microsurgery with handheld instruments," in Proceedings of the 26th International Conference of the Engineering in Medicine and Biology Society (EMBS), San Francisco, CA, USA, vol. 4, pp. 2771-2773, Sep. 2004

[16] M. A. Nasseri et al., "Clinical motion tracking and motion analysis during ophthalmic surgery using electromagnetic tracking system," in Proceedings of the 5th International IEEE Conference on BioMedical Engineering and Informatics (BMEI), Chongqing, China, pp. 10061010, Oct. 2012

[17] G. Saleh et al., "Evaluating surgical dexterity during corneal suturing," in Arch Ophthalmol, vol 124, issue 12, pp. 1263-1266, Sep. 2006

[18] J. Denavit and R.S. Hartenberg, "A kinematic notation for lower pair mechanisms based on matrices," in Transaction of the American Society of Mechanical Engineers (ASME). Journal of Applied Mechanics, vol. 77 , pp. $215-221,1955$ 